This article was downloaded by: [Agrillo, Christian]

On: 15 March 2010

Access details: Access Details: [subscription number 919880202]

Publisher Psychology Press

Informa Ltd Registered in England and Wales Registered Number: 1072954 Registered office: Mortimer House, 3741 Mortimer Street, London W1T 3JH, UK

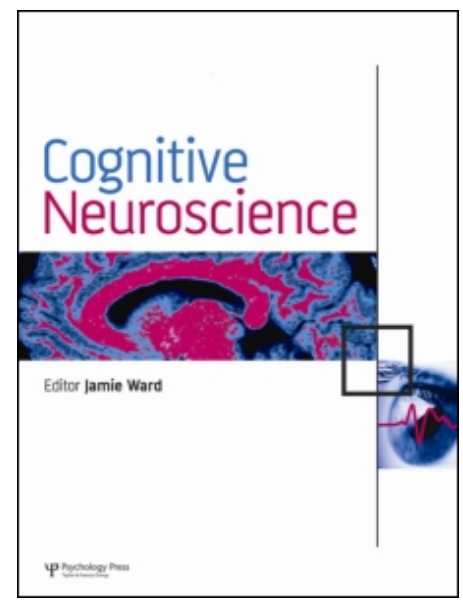

\title{
Cognitive Neuroscience
}

Publication details, including instructions for authors and subscription information:

http://www.informaworld.com/smpp/title $\sim$ content=t909559412

\section{Time and numerosity estimation are independent: Behavioral evidence for two different systems using a conflict paradigm}

Christian Agrillo ${ }^{\mathrm{a}}$; Ashish Ranpura ${ }^{\mathrm{b}}$; Brian Butterworth ${ }^{\mathrm{b}}$

${ }^{a}$ University of Padova, Padova, Italy ${ }^{b}$ University College London, London, UK

First published on: 15 March 2010

To cite this Article Agrillo, Christian, Ranpura, Ashish and Butterworth, Brian(2010) 'Time and numerosity estimation are independent: Behavioral evidence for two different systems using a conflict paradigm', Cognitive Neuroscience,, First published on: 15 March 2010 (iFirst)

To link to this Article: DOI: $10.1080 / 17588921003632537$

URL: http://dx.doi.org/10.1080/17588921003632537

\section{PLEASE SCROLL DOWN FOR ARTICLE}

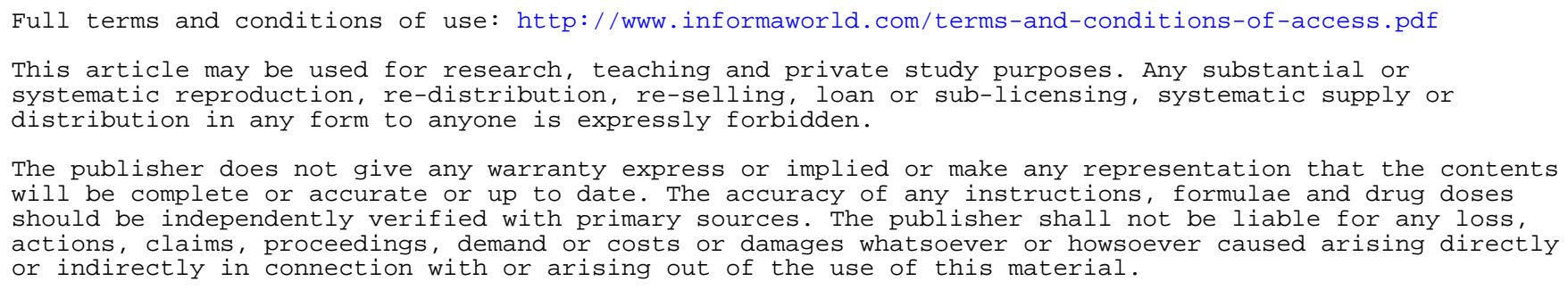




\title{
Time and numerosity estimation are independent: Behavioral evidence for two different systems using a conflict paradigm
}

\author{
Christian Agrillo \\ University of Padova, Padova, Italy \\ Ashish Ranpura and Brian Butterworth \\ University College London, London, UK
}

\begin{abstract}
Walsh (2003) proposed that time and numerical estimation are processed by one generalized magnitude system located mainly in the parietal cortex. According to this perspective, if the time and numerosity share the same mechanism, then interference effects should be observed when the two dimensions are put in conflict. In this study, 16 volunteers were required to listen to 25 audio files, differing in duration and number of tones, in two tasks: One required estimating the duration of the stimulus; the other required estimating the number of tones. For example, the same duration may contain $11,13,15,17$ or 19 tones, and 11 tones could last for $5,7,9,11$ or $13 \mathrm{~s}$. Results show that estimates of duration were unaffected by the number of tones, and estimates of numerosity were unaffected by duration: This is incompatible with time and numerosity being processed by the same mechanism. Theoretical implications are discussed.
\end{abstract}

Keywords: Numerical cognition; ATOM; Temporal and numerical estimation; Stroop paradigm.

\section{INTRODUCTION}

During the past decade a considerable body of experimental evidence has accumulated on the relation between time and number (Dormal, Seron, \& Pesenti, 2006; Vicario et al., 2008; Walsh, 2003; Xuan, Zhang, $\mathrm{He}$, \& Chen, 2007). According to classical models, organisms could quantify time and number simultaneously by using multiple switches and accumulators and the information about different magnitudes may hence be analyzed separately and compared or integrated according to metrics unique to each comparison (Meck \& Church, 1983). It has been proposed (Walsh, 2003) that time and number may be computed according to a common metric into a generalized magnitude system, and both numerical and temporal integration may be carried out by a distributed neural circuit activated by both timing and counting tasks. For instance, Brown (1997) provides behavioral evidence that time and number may share a single accumulator using dual tasks requiring both mental arithmetic and time estimation. Results showed that time tasks were disrupted but are not themselves good disrupters when no arithmetic was required as a secondary task, whereas performance decreased when mental arithmetic was performed together with time processing. Subsequently, it has also been demonstrated that the pattern of discrimination sensitivity to absolute (the size effect) and relative (the distance effect) difference is very similar in time and number (Pinel, Piazza, Le Bihan, \& Dehaene, 2004).

Xuan et al. (2007) reported an experiment where observers were required to judge the duration of the

Correspondence should be addressed to: Christian Agrillo, Department of General Psychology, University of Padova, via Venezia 8, I-35131 Padova, Italy. E-mail: christian.agrillo@unipd.it

The authors would like to thank Massimo Grassi and the two anonymous referees for their useful comments. This study was supported by research grants of the University of Padova. The reported experiments comply with all laws of the country (England) in which they were performed.

(C) 2010 Psychology Press, an imprint of the Taylor \& Francis Group, an Informa business www.psypress.com/cognitiveneuroscience 
stimuli while four types of nontemporal magnitude information (visual information such as number of dots, size, luminance of the elements, and numeric value of digits) were manipulated using a Strooplike paradigm. Results revealed that stimuli with larger magnitudes in these visual dimensions were judged to be temporally longer, suggesting that temporal and nontemporal dimensions may not be independent. More recently, Vicario et al. (2008) stated that the relation between time and number seems to be influenced by numerical information: Small numbers biased estimation toward short durations whereas large numbers biased estimation toward long durations in a behavioral standard time comparison task.

Further evidence on this hypothetical common system may come from comparative researches in which rats and pigeons were trained to discriminate auditory stimuli varying in both number and duration (i.e. two sounds in $2 \mathrm{~s}$ or eight sounds in $8 \mathrm{~s}$ ). After a preliminary shaping, subjects were tested in a transfer task, where novel stimuli varying in just one dimension were presented (two to eight tones in $4 \mathrm{~s}$ or four tones in 2-8 s): Results showed that animals spontaneously encoded information about both time and number; furthermore, the psychophysical function representing the probability that subjects categorized an intermediate value was virtually identical when time or number was varied (Meck \& Church, 1983).

Nonetheless, the exact relation between time and numerical information is still unclear and the idea that the two domains would be processed by one generalized magnitude system is not fully supported in the literature. There are not, for instance, clear anatomical correlates of the neural systems that would be involved in the two tasks. Even though many studies have reported that the parietal cortex is activated in aspects of temporal, spatial, and number processes (see Walsh, 2003), the exact location of this hypothetical unique system has not been identified.

Moreover, Dormal et al. (2006) have provided partial evidence of the independence of these domains. Comparisons of the duration and numerosity of series of flashing dots were tested in a Stroop design in which the two dimensions were controlled to create congruent, incongruent, and neutral pairs. Results showed that numerical cues interfered with the duration processing, facilitating duration processing when the numerosity and duration were congruent and interfering with it when they were incongruent. However, the authors also found that temporal cues did not affect numerosity processing: This leaves open the question as to whether time and number may be processed by different systems.
Although it has been argued that duration and numerosity are two "modes" of operation of the same accumulator mechanism, data from comparative studies demonstrate that many animals use numerical discrimination only as a "last-resort" strategy of quantification when other cues, such as time, cannot be used (Breukelaar \& Dalrymple-Alford, 1998). The quantification mechanism employed by animals is supposed to reflect the type of quantity information that is most relevant for the given context: In foraging situations, for instance, animals often attempt to maximize the amount of food acquired instead of the numerosity of pieces of food, and it has been demonstrated that rats trained to perform auditory sequences preferentially discriminate sequences on the basis of duration rather than number (Breukelaar \& Dalrymple-Alford, 1998). This leads us to propose that numerical and temporal information may be dissociated, since the two kinds of information were probably subject to different evolutionary pressures. Furthermore, Hobson and Newman (1981) reported different functions for the training curves in animals (e.g., linear functions for numerosity discrimination and power functions for time discrimination), suggesting that the two tasks may involve different processes.

The aim of the present paper is to investigate the relation between the estimation of time and of number by using a conflict paradigm requiring participants to estimate either the duration of the stimulus or the number of tones presented. If there is an interaction between number and time, this would provide evidence of a generalized mechanism underlying both domains, especially where there is conflict on the two dimensions. On the other hand, if performance is not affected by the task-irrelevant information (numerosity for Experiment 1 and time for Experiment 2), it will provide behavioral evidence for the independence of the two systems.

\section{METHOD}

\section{Participants}

A total of 16 volunteers (7 males and 9 females) between the ages of 24 and 31 (mean age 27.31) took part in the experiments. They were carried out at the Psychology Department of University College London.

\section{Stimuli}

Stimuli consisted of 25 different sound files (.wav format). The stimulus sequences were first created as 
MIDI files using Finale software (Allegro, 2007), which were then converted to audio files using Cool Edit Pro 2.1. Each stimulus consisted of an isochronous sequence of complex tones with the same pitch $(G)$, except that the first and last tones had another pitch (E) so that participants knew when each stimulus started and ended. The stimuli were presented at $75 \mathrm{~dB}$ SPL through headphones.

The number of tones and the duration of the stimuli were factorially combined. The stimuli had five different durations $(5,7,9,11$, and $13 \mathrm{~s})$ and, for each duration, five stimuli were prepared with $11,13,15$, 17, or 19 tones; six further stimuli, with identical features, were created for the training phase. Such stimuli, however, were not presented during the experiments. Matlab 6.5 software was used to create a specific program capable of presenting the stimuli according to the experimental design.

\section{Procedure}

Initially, a short familiarization training phase was presented. Participants listened to sample stimuli and tried to estimate either the duration or the number of tones in the stimulus. In the duration task, after the sequence had ended, participants held down the space bar as long as they estimated the whole duration of the stimulus (Figure 1); in the number task, the space bar was to be pressed as many times as participants estimated the number of tones presented. The same set of stimuli was presented in the duration and in the number task.

After training, the two tasks were presented in fixed blocks. Half of the participants performed the duration task first, and half performed the number task first. Instructions were provided before each block, so that participants knew which task they were going to perform before the presentation of the stimuli. No feedback was provided in either task.

\section{Analyses}

Both the experiments were analyzed using a $5 \times 5 \times 2$ Anova with Duration and Numerosity as within factors and Task order as between factors. The dependent variable was the distance between participants' response and the correct response, given by the formula: participants'

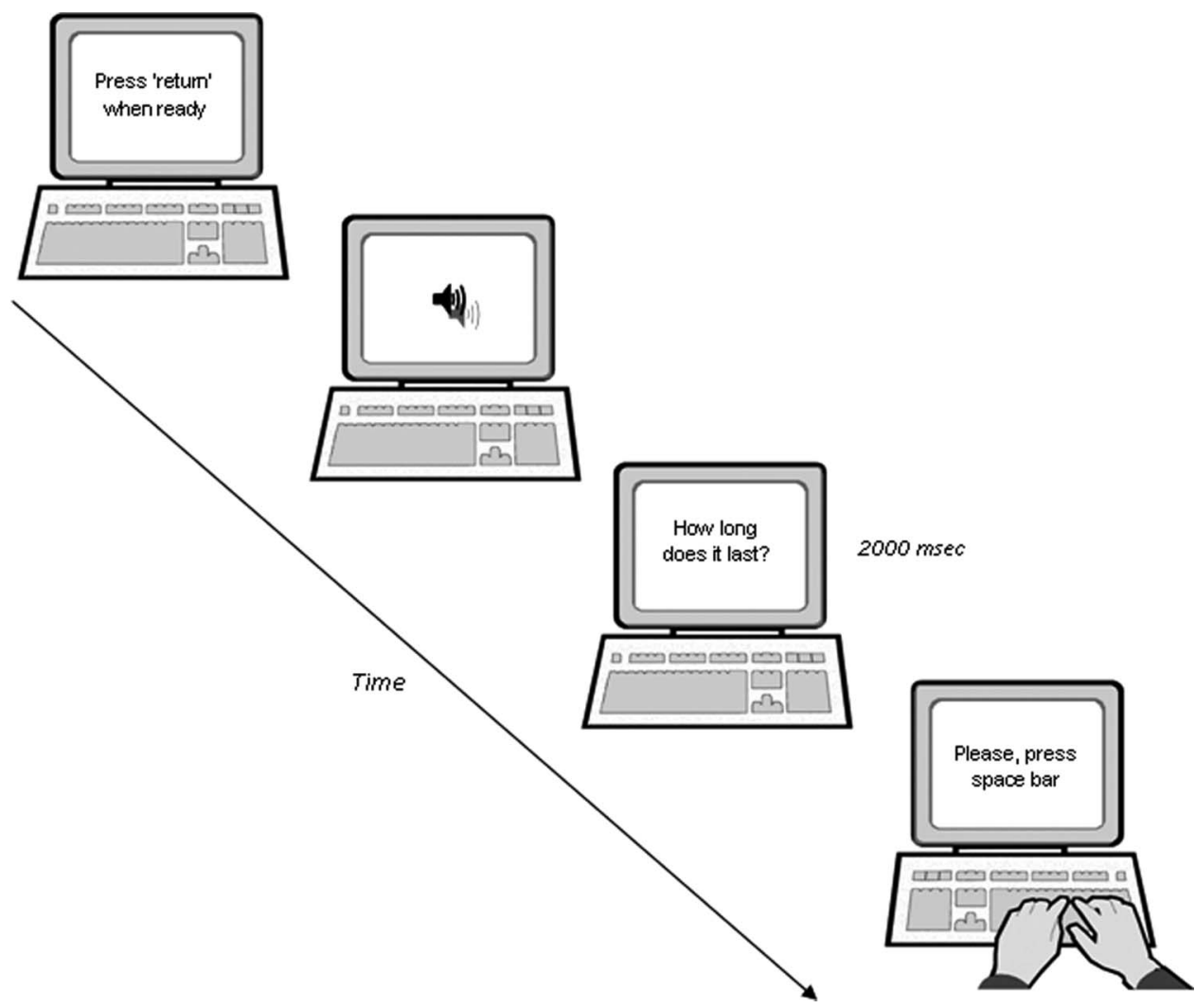

Figure 1. Experimental setting used in duration task. Number and duration tasks used the same stimuli. In the number task, the participant estimated the number of tones by repeatedly pressing the space bar on a computer keyboard; in the duration task, the participant estimated the duration of the whole stimulus by holding down the space bar. 
response - correct response. Data were transformed (square root) and, for all statistics, we took account of the Bonferroni correction.

\section{RESULTS}

\section{Experiment 1: Duration task}

There was a main effect of Duration, $F(4,56)=$ $15.874, p<.001$. No other main effect-Numerosity, $F(4,56)=2.224, p=.076$; Task order, $F(1,14)=$ $0.830, p=.378$ - or interaction was significant: Duration $\times$ Numerosity, $F(16,224)=1.653, p=.057$, Figure 2 a; Duration $\times$ Task order: $F(4,56)=0.553, p=.698$;
Numerosity $\times$ Task order, $F(4,56)=0.486, p=.746$; Duration $\times$ Numerosity $\times$ Task order, $F(16,224)=$ 1.435, $p=.127$. A likelihood ratio analysis (see Glover \& Dixon, 2004, for details) also reflected the absence of an interaction between Duration and Numerosity $(\lambda=2.44)$.

Overall there was a significant linear trend in underestimating the duration of the stimuli with increasing the duration, $F(1,79)=49.855, p<.01$. In detail, participants underestimated the longest duration-one-sample $t$-tests, $11 \mathrm{~s}, t(15)=-2.287, p=$ $.037 ; 13 \mathrm{~s}, t(15)=-7.945, p<.001$; while the other durations were more accurately estimated, $5 \mathrm{~s}, t(15)=$ $1.560, p=.140 ; 7 \mathrm{~s}, t(15)=-0.370, p=.716 ; 9 \mathrm{~s}, t(15)$ $=-0.191, p=.851$.
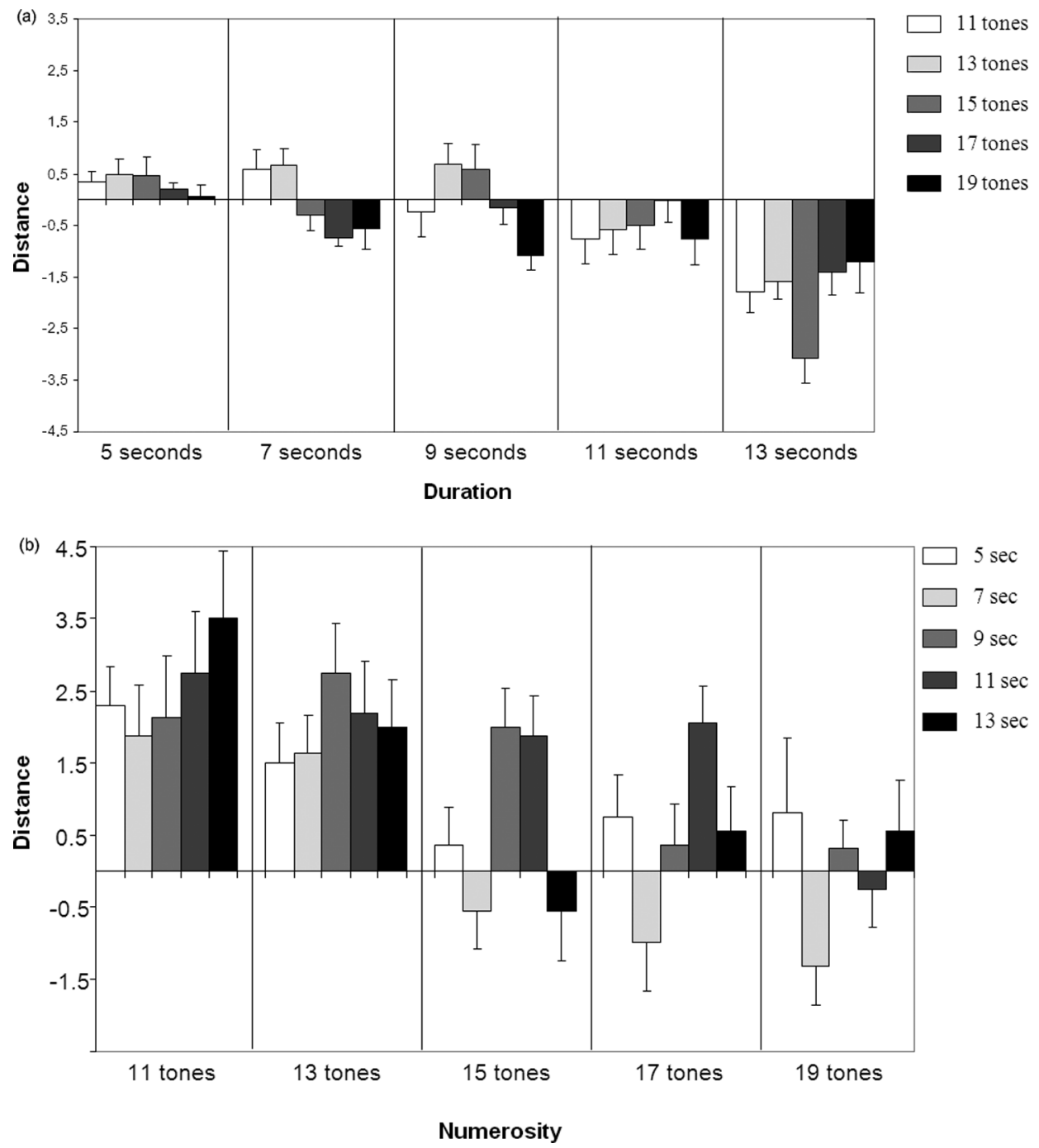

Figure 2. (a) Results of Experiment 1: distance (s) between the correct and the estimated duration for the different numbers of tones. (b) Results of Experiment 2: distance (number of tones) between the correct and the estimated number of tones for the different durations (untransformed data). 


\section{Experiment 2: Number task}

There was a main effect of Numerosity, $F(4,56)=$ $3.746, p=.009$. No other main effect-Duration, $F(4$, $56)=0.480, p=.750$; Task order, $F(1,14)=0.036$, $p=.853$ - or interaction was significant: Duration $\times$ Numerosity, $F(16,224)=1.043, p=.413$, Figure $2 b$; Numerosity $\times$ Task order, $F(4,56)=0.627, p=.645$; Duration $\times$ Task order, $F(4,56)=0.817, p=.520$; Numerosity $\times$ Duration $\times$ Task order, $F(16,224)=$ $1.208, p=.263$. A likelihood ratio analysis also reflected the absence of an interaction between Numerosity and Duration $(\lambda=1.78)$.

Overall there was a significant linear trend in which the overestimation of number of tones decreased with the number of tones presented, $F(1,79)=8.234, p=$ .005 . More specifically, participants overestimated the number of tones presented in the 11-tone condition, $t(15)=6.243, p<.001$, and the 13-tone condition, $t(15)=4.627, p<.001$, while they estimated the other conditions more accurately, 15 tones $t(15)=1.734, p=$ $.103 ; 17$ tones $t(15)=1.938, p=.072 ; 19$ tones $t(15)=$ $0.075, p=.942$.

\section{GENERAL DISCUSSION}

The present study aimed to investigate whether time and number rely on the same magnitude system. We therefore asked participants to estimate either the duration or the numerosity of the items in stimuli that were identical in the two tasks, thus putting the two dimensions in conflict. No statistical interaction between them was observed in either task. It was noteworthy that an analysis of the variances in the two tasks showed that the patterns of overestimation and underestimation in the two were different for time and for number. This is additional evidence that judgments of duration and number rely on two different systems, at least for the range of durations and numerosities studied here.

However, it is possible that several similar or even identical accumulators work simultaneously, so that numerosity and duration could be processed independently by two different accumulators before converging on a common system, as previously suggested (Oliveri et al., 2008). Alternatively, there may be separate stimulus-processing pathways: one for number-space and one for time-space. There is extensive evidence that number and space may share a common representation (a mental number line) and influence each other in tasks where they are processed together (e.g. Dehaene, Bossini, \& Giraux, 1993). Number can affect performance in several size estimation tasks (Calabria \& Rossetti, 2005; Kaufmann et al., 2005). Similarly, time and space have been reported to be processed together under some circumstances. DeLong (1981), for instance, found that the experience of time can be compressed together with space in scale-model environments, suggesting that spatial factors affect the perception of time.

The data from the present study do not contradict the interaction of space and number, nor of space and time, but they show potentially independent processing for numerosity and duration.

Conflict paradigms have been used to explore the interactions between two different domains or systems, here between time and number, and in particular to explore a possible shared mechanism for magnitudes (Pinel et al., 2004; Kaufmann et al., 2005; Xuan et al., 2007; Cohen Kadosh, Cohen Kadosh, \& Henik, 2008). Our results may appear inconsistent with those in Dormal et al. (2006), who found that in a duration comparison there was an interference from numerical cues, suggesting a shared mechanism. However, in their numerosity comparison task, temporal cues did not influence the numerosity judgements. One difference between our results and those of Dormal et al. (2006) occurred in the duration task, but they used visual stimuli (dots), while our study used auditory stimuli. It is possible, therefore, that time processing is affected by modality specificity, as suggested by brain imaging studies. For example, a recent transcranial magnetic stimulation (TMS) study provides compelling evidence for modality-specific timing. Bueti, Bahrami, and Walsh (2008) reported that, when judging the duration of a visual display, an increase in the difference threshold was observed on trials in which repetitive TMS was applied over V5/MT; on the other hand, consistent with a modality-specific assumption, no change in performance was found when subjects judged the duration of a tone. Similarly, modality specificity was observed in a functional magnetic resonance imaging (fMRI) study where participants were asked to tap a required rhythm, initially specified by either a visual or an auditory metronome (Jantzen, Steinberg, \& Kelso, 2005). Here activity remained high in area V5/MT after the metronome was terminated only in the visual condition.

The modality-specificity of duration processing supports the hypothesis of the intrinsic timing of temporal processing (Ivry \& Schlerf, 2008). According to these models, there is no specialized brain system for representing temporal information, since time is inherent in the neural dynamics. It has been proposed that this property might either be limited to neural regions that are capable of sustaining their activity in the absence of sensory input (Reutimann, Yakovlev, Fusi, \& Senn, 2004) or, alternatively, be ubiquitous, and arise as part of modality-specific processing (Burr, Tozzi, \& Morrone, 2007). 
The existence of two independent systems for time and number is also supported by the evidence of different anatomical correlates in temporal and numerical estimation tasks. It has been argued that specific neural regions might serve in timing systems, such as the basal ganglia (Rao, Mayer, \& Harrington, 2001), supplementary motor area (Macar, Coull, \& Vidal, 2006) and prefrontal cortex, especially in the right hemisphere (Lewis \& Miall, 2006) and there is little evidence for parietal interval timing. On the other hand, numerosity tasks seem to involve mainly parietal areas such as intraparietal sulcus (Piazza, Izard, Pinel, Le Bihan, \& Dehaene, 2004).

In conclusion, the present research provided behavioral evidence for the independence of number and time processing. Castelli, Glaser, and Butterworth (2006) reported a distinct neural activation to compute discrete (numerical information) and analog quantities (continuous variables such as cumulative surface area), therefore it is possible that time and continuous quantity share a magnitude system and will interact with each other. Further studies where analog quantities will be orthogonally manipulated with duration are needed to investigate this aspect.

Manuscript received 20 October 2009 Manuscript accepted 11 January 2010 First published online

\section{REFERENCES}

Breukelaar, J. W., \& Dalrymple-Alford, J. C. (1998). Timing ability and numerical competence in rats. Journal of Experimental Psychology: Animal Behavior, 24, 84-97.

Brown, S. W. (1997). Attentional resources in timing: Interference effects in concurrent temporal and non-temporal working memory tasks. Perception \& Psychophysics, 59, 1118-1140.

Bueti, D., Bahrami, B., \& Walsh, V. (2008). Sensory and association cortex in time perception. Journal of Cognitive Neuroscience, 20(6), 1054-1062.

Burr, D., Tozzi A., \& Morrone, C. M. (2007). Neural mechanisms for timing visual events are spatially selective in real-world coordinates. Nature Neuroscience, 10, 423-425.

Calabria, M., \& Rossetti, Y. (2005). Interference between number processing and line bisection: A methodology. Neuropsychologia, 43, 779-783.

Castelli, F., Glaser, D. E., \& Butterworth, B. (2006). Discrete and analogue quantity processing in the parietal lobe: A functional MRI study. Proc Nat Acad USA, 103(12), 4693-4698.

Cohen Kadosh, R., Cohen Kadosh, K., \& Henik, A. (2008). When brightness counts: The neuronal correlate of numerical-luminance interference. Cerebral Cortex, 8, 337-343.

Dehaene, S., Bossini, S., \& Giraux, P. (1993). The mental representation of parity and numerical magnitude. Jnl Exp Psychology: General, 122, 371-396.
DeLong, A. J. (1981). Phenomenological space-time: Towards an experiential relativity. Science, 213, 681-683.

Dormal, V., Seron, X., \& Pesenti, M. (2006). Numerosityduration interference: A Stroop experiment. Acta Psychologica, 121, 109-124.

Glover, S., \& Dixon, P. (2004). Likelihood ratios: A simple and flexible statistic for empirical psychologists. Psychonomic Bulletin \& Review, 11, 791-807.

Hobson, S. L., \& Newman, F. (1981). Fixed-ratio counting schedules: Response and time measures considered. In M. L. Commons \& J. A. Nevin (Eds.), Quantitative analyses of behavior: Discriminative properties of reinforcement schedules (pp. 193-224). Cambridge, MA: Ballinger.

Ivry, R. B., \& Schlerf, J. E. (2008). Dedicated and intrinsic models of time perception. Trends in Cognitive Sciences, 12(7), 273-280.

Jantzen, K. J., Steinberg F. L., \& Kelso, J. A. S. (2005). Functional MRI reveals the existence of modality and coordination-dependent timing networks. NeuroImage, 25, 1031-1042.

Kaufmann, L., Koppelstaetter, F., Delazer, M., Siedentopf, C., Rhomberg, P., Golaszewski, S., et al. (2005). Neural correlates of distance and congruity effects in a numerical Stroop task: An event-related fMRI study. NeuroImage, 25, 888-898.

Lewis, P. A., \& Miall, R. C. (2006). A right hemispheric prefrontal system for cognitive time measurement. Behavioural Processes, 71, 226-234.

Macar, F., Coull, J., \& Vidal, F. (2006). The supplementary motor area in motor and perceptual time processing: fMRI studies. Cognitive Processes, 7, 89-94.

Meck, W. H., \& Church, R. M. (1983). A mode control model of counting and timing processes. Jnl Exp Psychology: Animal Behavior Processes, 9(3), 320-334.

Oliveri, M., Vicario, C. M, Salerno, S., Koch, G., Turriziani, P., Mangano, R., et al. (2008). Perceiving numbers alters time perception. Neuroscience Letters, 438(3), 308-311.

Piazza, M., Izard, V., Pinel, P., Le Bihan, D., \& Dehaene, S. (2004). Tuning curves for approximate numerosity in the human intraparietal sulcus. $\mathrm{Neu}$ ron, 44, 547-555.

Pinel, P., Piazza, M., Le Bihan, D., \& Dehaene, S. (2004). Distributed and overlapping cerebral representations of number, size, and luminance during comparative judgments. Neuron, 41, 983-993.

Rao, S. M., Mayer, A. R., \& Harrington, D. L. (2001). The evolution of brain activation during temporal processing. Nature Neuroscience, 4, 317-323.

Reutimann, J., Yakovlev, V., Fusi S., \& Senn, W. (2004). Climbing neuronal activity as an event based cortical representation of time. Jnl Neuroscience, 24, 3295-3303.

Vicario, C. M., Pecoraro, P., Turriziani, P., Koch, G., Caltagirone C., \& Oliveri, M. (2008). Relativistic compression and expansion of experiential time in the left and right space. PLOS ONE 3(3), e1716.

Walsh, V. (2003). A theory of magnitude: Common cortical metrics of time, space and quantity. Trends in Cognitive Sciences, 7(11), 483-488.

Xuan, B., Zhang, D., He, S., \& Chen, X. (2007). Larger stimuli are judged to last longer. Jnl of Vision, 7, 1-5. 